\title{
Music in the Treatment of Affective Disorders: An Exploratory Investigation of a New Method For Music-Therapeutic Research
}

\author{
Stefan Koelsch \\ Freie Universität Berlin, Berlin, Germany \\ Kristin Offermanns and Peter Franzke \\ Max Planck Institute for Human Cognitive \\ Neuroscience, Leipzig, Germany
}

MUSIC LISTENING AND MAKING ACTIVATES A multitude of brain structures, the engagement of which is likely to have beneficial effects on the psychological and physiological health of individuals. We first briefly review functional neuroimaging experiments on music and emotion, showing that music-evoked emotions can change activity in virtually all core areas of emotional processing. We then enumerate social functions that are automatically and effortlessly engaged when humans make music. Engagement in these social functions fulfils basic human needs, is part of what makes us human, and is an important source for pleasure and happiness. Finally, we present a new method for music therapy, including an exploratory empirical study on effects of music making. Results show that the music making increased the mood of individuals compared to a control group. This music therapy method is promising in encouraging further development for the treatment of affective disorders, and can be used in both single- and double-blinded studies for empirical, evidence-based medical research.

Received November 11, 2009, accepted January 26, 2010.

Key words: music therapy, neuroscience, emotion, social cognition, depression

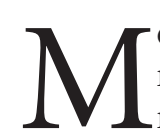

OUNTING EVIDENCE INDICATES THAT making music, dancing, and even simply listening to music activates a multitude of brain structures involved in cognitive, sensorimotor, and emotional processing (Koelsch \& Siebel, 2005; Koelsch, Siebel, \& Fritz, 2010; Zatorre, Chen, \& Penhune, 2007). However, there is still lack of systematic high-quality research investigating possible beneficial effects of the engagement of such processes on the psychological and physiological health of individuals.

\section{Limbic and Paralimbic Correlates of Music-Evoked Emotions}

In most humans, music can strongly affect emotion and mood, and such effects are among the main reasons to produce, and listen to, music (for a review see Juslin \& Västfjäll, 2008). Functional neuroimaging and lesion studies have shown that music-evoked emotions involve core structures of emotional processing (as will be reviewed below), arguing for the notion that musical emotions are real emotions (and not simply illusions), and biologically comparable to everyday emotions (for reviews see Juslin \& Västfjäll, 2008; Koelsch, 2010; Koelsch et al., 2010). Such emotional effects form an important basis for a possible intervention using music in the treatment of disorders related to autonomic, endocrine, and immune system dysfunction, because the activity of these systems is under modulatory control of emotional processes (Dantzer, O'Connor, Freund, Johnson, \& Kelley, 2008; Koelsch \& Siebel, 2005). This section will review functional neuroimaging studies on music and emotion, illustrating that activity of core structures of emotional processing can be modulated by music-evoked emotions (for a review on endocrine and immunological effects of music listening, music making, and dance, see Quiroga Murcia, Kreutz, \& Bongard, in press).

With regard to emotional processing, previous functional neuroimaging studies have shown that listening to music can have effects on the activity of virtually all limbic and paralimbic structures (that is, of core structures of emotional processing), in both musicians and in so-called nonmusicians. In a seminal PET experiment, Blood and Zatorre (2001) used naturalistic music to evoke strongly pleasurable experiences involving "chills" or "shivers down the spine." Increasing chills intensity correlated with increases in regional cerebral blood flow ( $\mathrm{rCBF}$ ) in brain regions thought to be involved in reward and emotion, including the ventral striatum (presumably the nucleus accumbens, NAc), the insula,

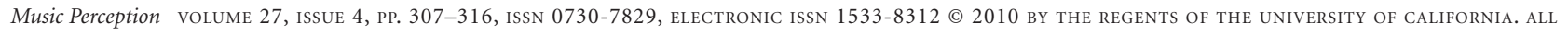
RIGHTS RESERVED. PLEASE DIRECT ALL REQUESTS FOR PERMISSION TO PHOTOCOPY OR REPRODUCE ARTICLE CONTENT THROUGH THE UNIVERSITY OF CALIFORNIA PRESS'S RIGHTS AND PERMISSIONS WEBSITE, HTTP://WWW.UCPRESSJOURNALS.COM/REPRINTINFO.ASP. DOI:10.1525/MP.2010.27.4.307 
anterior cingulate cortex (ACC), orbitofrontal cortex, and ventral medial prefrontal cortex. The authors also found decreases in $\mathrm{rCBF}$ in the amygdala as well as in the hippocampus with increasing chills intensity. Thus, activity changes were observed in central structures of the limbic/paralimbic system (e.g., amygdala, NAc, ACC, and hippocampus). This was the first study showing modulation of amygdalar activity with music, which was important for two reasons: First, it supported the assumption that music can evoke "real" emotions and that, thus, emotions evoked by music are not mere illusions, because the activity of core structures of emotion processing was modulated by music (for details, see Koelsch et al., 2010). Second, it strengthened the empirical basis for music-therapeutic approaches for the treatment of affective disorders such as depression and pathologic anxiety, because these disorders are partly related to amygdalar dysfunction (Drevets et al., 2002; Stein, Simmons, Feinstein, \& Paulus, 2007).

An fMRI study from our group (Koelsch et al., 2006) showed that activity changes in the amygdala, ventral striatum, and hippocampus can be evoked by music even without individuals having intense "chill" experiences. That study (Koelsch, Fritz, von Cramon, Müller, \& Friederici, 2006) compared brain responses to joyful instrumental tunes (played by professional musicians) with responses to electronically manipulated, continuously dissonant counterparts of these tunes. Unpleasant music elicited increases in blood-oxygen-level dependent (BOLD) signals in the amygdala, hippocampus, parahippocampal gyrus, and temporal poles (and decreases of BOLD signals were observed in these structures in response to the pleasant music). During the presentation of the pleasant music, increases of BOLD signals were observed in the ventral striatum (presumably NAc) and the insula (in addition to some cortical structures not belonging to limbic or paralimbic circuits that will not be further reported here). In addition to the mentioned studies from Blood and Zatorre (2001) and Koelsch et al. (2006), several other functional neuroimaging studies (Ball et al., 2007; Baumgartner, Lutz, Schmidt, \& Jäncke, 2006; Eldar, Ganor, Admon, Bleich, \& Hendler, 2007; Koelsch, Sammler, Jentschke, \& Siebel, 2008; Lerner, Papo, Zhdanov, Belozersky, \& Hendler, 2009) and lesion studies (e.g., Gosselin, Peretz, Johnsen, \& Adolphs, 2007) showed involvement of the amygdala in emotional responses to music.

Compared to studies investigating neural correlates of emotion with stimuli other than music (e.g., photographs with emotional valence, or stimuli that reward or punish the subject), the picture provided by functional neuroimaging studies on music and emotion bears a particularly striking feature: The number of studies reporting activity changes within the (anterior) hippocampal formation (Blood \& Zatorre, 2001; Baumgartner et al., 2006; Eldar et al., 2007; Koelsch et al., 2006; Mitterschiffthaler, Fu, Dalton, Andrew, \& Williams, 2007) is remarkably high. We (Koelsch et al., 2010) have previously argued that the hippocampus (perhaps particularly the anterior hippocampal formation) plays an important role for the generation of tender positive emotions (such as joy and happiness), and, in our view, one of the great powers of music is to evoke hippocampal activity related to such emotions (see also Koelsch, in press, for details). The activity changes in the (anterior) hippocampal formation evoked by listening to music are relevant for music therapy because patients with depression or post-traumatic stress disorder (PTSD) show a volume reduction of the hippocampal formation (associated with a loss of hippocampal neurons and blockage of neurogenesis in the hippocampus; Warner-Schmidt \& Duman, 2006), and individuals with reduced capability of producing tender positive emotions show reduced activity changes in the hippocampus in response to music (Koelsch et al., 2007). Therefore, it could be hypothesized that music therapy can help to: (a) reanimate activity in the hippocampus, (b) prevent death of hippocampal neurons, and (c) lift the blockage of hippocampal neurogenesis.

Similarly, because the amygdala and the NAc function abnormally in patients with depression, studies showing modulation of activity within these structures lend to the hypothesis that music can be used to modulate activity of these structures (either by listening to or by making music), and thus ameliorate symptoms of depression. The scientific evidence for effectiveness of music therapy on depression is surprisingly weak, perhaps due to the lack of high quality studies, and the small number of studies with randomized, controlled trials (for an overview, see Maratos, Gold, Wang, \& Crawford, 2008).

\section{Social Functions of Music: The Seven Cs}

The former section illustrated that music can activate brain structures involved in reward and pleasure (such as the nucleus accumbens). This section will provide some explanations as to why (preferred) music is perceived as rewarding and pleasurable. Human music making is an activity involving several social functions, which we propose here to divide into seven different areas. The ability and need to practice these social functions is part of what makes us human, and emotional effects of engaging in these functions include joy and happiness (such effects have important implications for music 
therapy). Exclusion from the engagement in these functions represents an emotional stressor, and has deleterious effects on health (Cacioppo \& Hawkley, 2003). Therefore, engaging in such social functions is important for the survival of the individual and thus for the human species. In the following, we will outline seven different dimensions of social functions.

(1) When we make music, we make contact with other individuals. Being in contact with other individuals is a basic need of humans (as well as of numerous other species; Harlow, 1958), and social isolation is a major risk factor for morbidity as well as mortality (Cacioppo \& Hawkley, 2003; House, Landis, \& Umberson, 1988).

(2) Music automatically engages social cognition (Steinbeis \& Koelsch, 2009). During music listening, individuals automatically engage processes of mental state attribution ("mentalizing" or "adopting an intentional stance") in an attempt to figure out the intentions, desires, and beliefs of the individuals who actually created the music (also often referred to as establishing a "theory of mind," TOM). A recent fMRI study (Steinbeis \& Koelsch, 2009) investigated whether listening to music would automatically engage a TOM-network (typically comprising anterior frontomedian cortex, temporal poles, and the superior temporal sulcus). In that study we presented non-tonal music (from Arnold Schönberg and Anton Webern) to nonmusicians, either with the cue that they were written by a composer or with the cue that they were generated by a computer. Participants were not informed about the experimental manipulation, and the task was to rate after each excerpt how pleasant or unpleasant they found each piece to be. A post-imaging questionnaire revealed that during the Composer condition, participants felt more strongly that intentions were expressed by the music (compared to the Computer condition). Correspondingly, the fMRI data showed that during the Composer condition (contrasted to the Computer condition) BOLD signals increased strongly in precisely the neuroanatomical network dedicated to mental state attribution, namely the anterior medial frontal cortex (aMFC), the left and right superior temporal sulcus, as well as left and right temporal poles. Notably, the brain activity in the aMFC correlated with the degree to which participants thought that an intention was expressed in the composed pieces of music. This study thus showed that listening to music automatically engages areas dedicated to social cognition (i.e., a network dedicated to mental state attribution in the attempt to understand the composer's intentions).

(3) Music making can engage co-pathy in the sense that interindividual emotional states become more homogenous (e.g., reducing anger in one individual and depression or anxiety in another), thus decreasing conflicts and promoting cohesion of a group (e.g., Huron, 2001). With regard to positive emotions, for example, co-pathy can increase the well-being of individuals during music making or during listening to music. We use the term "copathy" here (instead of "empathy") because empathy has many different connotations, due to various definitions of empathy provided by different researchers. By using the term co-pathy we not only refer to the phenomenon of thinking what one would feel if one were in someone else's position, but we refer to the phenomenon that one's own emotional state is actually affected in the sense that it occurs when one perceives (e.g., observes or hears) or imagines someone else's affect, and this evokes a feeling in the perceiver that bears strong congruency with what the other individual is feeling (see also Singer \& Lamm, 2009). Co-pathy should be differentiated from: (a) mimicry (a low-level perception-action mechanism that may contribute to empathy); (b) emotional contagion (a precursor of empathy; e.g. children laughing because other children laugh)—both mimicry and emotional contagion may occur outside of awareness and do not require a self/other concept; (c) sympathy, empathic concern, and compassion (which do not necessarily involve shared feelings; e.g., feeling pitiful for a jealous person without feeling jealous oneself; see Singer \& Lamm, 2009). Thus, co-pathy requires self awareness and self/other distinction; that is, the capability to make oneself aware that the affect may have been evoked by music made by others, although the actual source of one's affect lies within oneself.

(4) Music always involves communication. Notably, for infants and young children, musical communication during parent-child singing of lullabies and play songs is important for social and emotional regulation, as well as for social, emotional, and cognitive development (Fitch, 2006; Trehub, 2003). Neuroscience and behavioral studies have revealed considerable overlap between the neural substrates and cognitive mechanisms underlying the processing of musical syntax and language syntax (Koelsch, 2005; Patel, 2007; Steinbeis \& Koelsch, 2008). Moreover, musical information can systematically influence semantic processing of language (Koelsch et al., 2004; Steinbeis \& Koelsch, 2009). It is also worth noting that the neural substrates engaged in speech and song strongly overlap (Callan et al., 2006). Because music is a means of communication, particularly active music therapy (in which patients make music) can be used to train skills of (nonverbal) communication (Hillecke, Nickel, \& Bolay, 2005).

(5) Music making also involves coordination of actions. This requires the ability to synchronize to a beat and keep 
a beat, a human capability that is unique among primates (although other species are capable of synchronizing movements to an external beat as well; Patel, Iversen, Bregman, \& Schulz, 2009). The coordination of movements in a group of individuals appears to be associated with pleasure (for example, when dancing together), even in the absence of a shared goal (apart from deriving pleasure from concerted movements; see also Huron, 2001). Interestingly, a recent study from Kirschner and Tomasello (2009) reported that children as young as $2^{1 / 2}$ years synchronized more accurately to an external drum beat in a social situation (i.e., when the drum beat was presented by a human play partner) compared to non-social situations (i.e., when the drum beat was presented by a drumming machine, or when the drum sounds were presented via a loudspeaker). This effect might have originated from the pleasure that emerges when humans coordinate movements between individuals (Overy \& Molnar-Szakacs, 2009; Wiltermuth \& Heath, 2009).

(6) A sound musical performance by multiple players is only possible if it also involves cooperation between players. Cooperation involves a shared goal, and engaging in cooperative behavior is an important potential source of pleasure. For example, Rilling et al. (2002) reported an association between cooperative behavior and activation of a reward network including the nucleus accumbens. Cooperation between individuals increases interindividual trust and increases the likelihood of further cooperation between these individuals. It is worth noting that only humans have the capability to communicate about coordinated activities during cooperation to achieve a joint goal (Tomasello, Carpenter, Call, Behne, \& Moll, 2005).

(7) As an effect, music leads to increased social cohesion of a group (Cross \& Morley, 2008). A wealth of studies have shown that humans have a "need to belong" and a strong motivation to form and maintain enduring interpersonal attachments (Baumeister \& Leary, 1995). Meeting this need increases health and life expectancy (Cacioppo \& Hawkley, 2003). Social cohesion also strengthens the confidence in reciprocal care (see also the caregiver hypothesis; Fitch, 2005), and the confidence that opportunities to engage with others in the mentioned social functions also will emerge in the future.

Although it should clearly be noted that music also can be used to manipulate other individuals and to support non-social behavior (e.g., Brown \& Volgsten, 2006), music is still special-although not unique-in that it can engage all of these social functions at the same time, which is presumably one explanation for the emotional power of music (for a discussion on the role of other factors, such as sexual selection, and for the evolution of music see Fitch, 2005; Huron, 2001). Therefore, music does serve the goal to fulfil social needs (our need to be in contact with others, to belong, to communicate, etc.). In this regard, music-evoked emotions are related to survival functions and to functions that are of vital importance for the individual. Now we will try to integrate the neural basis of music-evoked emotions and the social functions of music making by suggesting a new method for music therapeutic intervention research.

\section{An Exploratory Investigation on a New Method for Music-Therapeutic Research}

The previous sections illustrated (a) that music is capable of modulating activity in virtually all limbic and paralimbic brain structures, (b) that pleasant music can modulate neural systems involved in reward and pleasure, and (c) that the power of music to motivate individuals to engage vital social functions is an important source of pleasure. These observations motivated us to investigate effects of music making on mood, a topic that has, surprisingly, only sparsely been addressed so far (for an overview, see e.g. Kreutz, Bongard, Rohrmann, Hodapp, \& Grebe, 2004; for positive effects of dancing to music on mood see Quiroga Murcia, Bongard, \& Kreutz, 2009). A previous study from Kreutz et al. (2004) provides data showing that mood (as measured with the Positive and Negative Affect Schedule) increased during singing compared to listening to music. Other studies on this topic suggest similar outcomes, but face methodological difficulties such as lack of a control group (Bittman et al., 2005; Bittman, Bruhn, Stevens, Westengard, \& Umbach, 2003; Bittman et al., 2004) or rather small sample sizes ( $n \leq 10$; Grape, Sandgren, Hansson, Ericson, \& Theorell, 2003; Valentine \& Evans, 2001; Waldon, 2001). In the following, we will report an investigation on the effects of joint music making on the mood of individuals, and introduce a new music therapy method in which participants play along in groups of two to three participants to joyful music played from a stereo system. The rationale of this method was that music-evoked emotions (partly due to the engagement in social functions during music making) evoke positive emotions and improve the mood of individuals. To compare effects of music making, we also measured a control group, in which participants performed a movement task in time with the beat of a matched auditory control stimulus.

We used the Profile of Mood States (POMS) to assess mood before and after the experimental session with the hypothesis that mood measures (as indicated by the four scales of the POMS: depression/anxiety, vigor, fatigue, and irritability) would improve in the music as opposed 
to the control group. We also obtained data on the emotional state of participants during the experiment to assess the emotional impact of the procedure.

\section{Method}

\section{Participants}

Our study population comprised 154 participants (none of them had more than three years of formal music training, all of them considered themselves as nonmusicians). Eighty-one of these participants were randomly assigned to a music group (mean age 24.4 years, range 19-31 years, 42 females and 39 males), and 73 individuals to the control group (mean age 24.9 years, age range 18-31 years, 36 females and 37 males).

\section{Stimuli}

The musical stimulus consisted of 18 pieces from various epochs and styles (e.g., Classic, Jazz, Irish folk, Salsa, Andean music, Reggae; see Appendix for list of pieces); the range of beats per minute (BPM) was 106-132. All pieces were instrumental music (i.e., music without lyrics), and were judged by a music psychologist as happy.

The control stimulus consisted of 18 computergenerated pieces that were isochronous sequences of random tones (monophonic) of the chromatic scale. That is, all pitch classes from the chromatic scale occurred with equal probability $(p=1 / 12)$, and no major-minor tonal centre was established. Control stimuli matched the music pieces with regard to pitch range, mean pitch, tempo (BPM), and duration. Tones of each control piece were synthesized with Reason 3.0 (Propellerhead Software, Stockholm/Sweden), using timbres that were reminiscent of the respective experimental musical stimulus. Each stimulus set (music, control pieces) had a duration of approximately 41 minutes.

\section{Experimental Setup and Procedure}

Each experimental session was performed with three participants (if one of the three participants did not show up, s/he was substituted by an experimenter; this was the case in about twelve percent of the sessions, with no difference between the experimental and control groups). During the experiment, participants were separated by blinds (so that they could hear but not see each other).

In the music group, each participant had a set of instruments consisting of drum (Djembe), tambourine, percussion instruments, and a pentatonic xylophone.
During the presentation of the music, participants were asked to play along to the music with their instruments in a way that they liked most, and in a way that the others would hear him/her (this was also ascertained by the experimenter). Participants had the option to change instruments whenever they liked.

In the control group, each participant had a clothcovered fibre board with five circles printed in one row on top of them. Participants could choose between two different sticks ( $25 \mathrm{~cm}$ long, and 2 or $3 \mathrm{~cm}$ in diameter) and were asked to tap the sticks in time with the beat of the auditory stimulus into the circles (from left to right) in a way that the others could hear him/her (as for the music group, this was also ascertained by the experimenter; thus, the experimenter also was able to ensure the compliance of participants). Participants had the option to change sticks whenever they liked.

Thus, both groups: (a) were presented with an auditory stimulus comparable in tempo as well as pitch (and partly also with regards to timbre), (b) carried out movement tasks, (c) synchronized to the auditory stimulus, and (d) participated in the experiment within a group situation. Both groups were independent; that is, no-one participated in both groups (and each participant performed the experiment only once).

In both groups, every piece was followed by a $20 \mathrm{~s}$ silent interval in which participants made ratings on how they felt during the last piece in terms of valence, arousal, and six basic emotions (happy, angry, disgusted, anxious, sad, surprised). Valence and arousal ratings were obtained using modified (9-point) selfassessment manikins (SAMs; Bradley \& Lang, 1994). Basic emotion ratings were obtained using Likert scales ranging from 0 ("not at all") to 8 ("very strongly").

Before and after the music sessions, participants filled out the short (35-item) German version of the Perception Of Mood States (POMS; Biehl, Dangel, \& Reiser, 1986), a measure of psychological distress consisting of the four scales "Depression/Anxiety," "Fatigue," "Vigor," and "Irritability." Before the experiment, participants filled out the Toronto Alexithymia Scale (TAS-26; Kupfer, Brosig, \& Brähler, 2001). We also obtained rest electrocardiograms (ECGs) and blood pressure before and after the experimental sessions, personality questionnaires before the sessions, and salivary samples as well as ECG data during the sessions. However, for the sake of brevity those data will be reported elsewhere.

\section{Data Evaluation}

Participants with a TAS-26 total score of 54 or above (indicative for alexithymia as according to the manual 
TABLE 1. Behavioral Data Obtained From the Profile of Mood States (POMS).

\begin{tabular}{|c|c|c|c|c|c|c|c|}
\hline \multirow[b]{2}{*}{ POMS Scale } & \multicolumn{2}{|c|}{ Music Group } & \multicolumn{2}{|c|}{ Control Group } & \multicolumn{2}{|c|}{ Group Difference } & \multirow[b]{2}{*}{ Interaction } \\
\hline & Pre & Post & Pre & Post & Pre & Post & \\
\hline Depression & $6.57(8.01)$ & $3.51(5.91)$ & $8.25(10.37)$ & $9.3(9.68)$ & 2732.50 & $1701.50^{* * *}$ & $14.50^{* *}$ \\
\hline Fatigue & 9.75 (7.08) & 7.38 (6.95) & $12.32(7.35)$ & $15.15(8.75)$ & 2300.00 & $1387.50^{\star * *}$ & $14.06^{\star *}$ \\
\hline Vigor & $21.65(7.12)$ & $25.32(8.50)$ & $20.81(6.54)$ & $17.11(6.79)$ & 2791.00 & $1289.50^{* * *}$ & $23.12^{\star \star *}$ \\
\hline Irritability & $2.26(3.82)$ & $1.95(3.52)$ & $3.12(5.23)$ & $8.10(7.55)$ & 2682.00 & $1282.00^{\star * *}$ & $26.77^{\star * *}$ \\
\hline
\end{tabular}

Note: The four left columns show means (with SD in parentheses) of pre and post session measurements for the Music and Control groups. The fifth and sixth columns show Mann-Whitney $U$ values to test group differences separately for pre and post measures ( $n=81$ for the experimental group; $n=73$ for the control group). Significant $p$ values are indicated by asterisks $\left({ }^{* *} p<.0001,{ }^{* *} p<.001\right)$. The final column shows the chi-square values of Kruskal-Wallis non-parametric ANOVAs testing the interaction between pre/post measures and group $(d f=1)$.

(Kupfer et al., 2001) were excluded (this was the case for twelve individuals of an initial sample of $n=166$, leading to the study population of $n=154$ ). The 9-point scales for valence and arousal were recoded into an arousal scale ranging from 0 ("very relaxed") to 8 ("very excited"), and a valence scale ranging from-4 ("very unpleasant") to 4 ("very pleasant").

Statistical analysis was performed using SPSS 16.0. Non-parametric tests were used because some variables were not normally distributed, or showed inhomogeneity of variance: Group differences for pre and post measures of the POMS were calculated with two-sided, independent samples Mann-Whitney $U$ tests; interactions between the factors pre/post measures and group were calculated with Kruskal-Wallis non-parametric ANOVAs ( $p$ values were Bonferroni-corrected).

\section{Results}

\section{POMS Data}

Data obtained from the POMS (Profile of Mood States) before the experimental sessions did not differ between groups on any of the four POMS scales (Table 1). By contrast, groups differed on all four scales after the experiment, with the interaction between pre/post measures and group being significant for all scales (Table 1). A comparison of pre and post measures (using Wilcoxon Signed Rank tests, with $n=81$ for the experimental group, and $n=73$ for the control group) furthermore showed that (a) depression/anxiety did not change in the control group $(\mathrm{Z}=-0.98, p=.33)$, but decreased in the music group $(Z=-5.46, p<.0001)$, (b) fatigue tended to increase in the control group $(Z=-2.31, p<.03)$, but decreased in the music group $(Z=-2.98, p<.003)$, (c) vigor decreased in the control group $(Z=-3.36$, $p<.001)$ but increased in the music group $(Z=-3.46$, $p<.001)$, and (d) irritability increased in the control group $(Z=-4.76, p<.0001)$ but did not change in the music group $(\mathrm{Z}=-0.62, p=.54)$.

\section{Valence, Arousal, and Emotion Ratings}

During the experiment, music group participants felt more pleasant, more aroused, happier, less angry, less sad, and less anxious compared to the control group (Table 2). The largest difference in emotion ratings was observed for happiness ratings $(M=4.69$ in the music

TABLE 2. Emotion Measure Means (SDs) for the Music and Control Groups.

\begin{tabular}{lccc}
\hline Emotion Measure & Music Group & Control Group & Group Difference \\
\hline Valence $^{1}$ & $1.90(0.95)^{2}$ & $0.10(0.92)^{3}$ & $484.50^{\star * *}$ \\
Arousal $^{4}$ & $4.50(1.15)$ & $3.99(1.23)$ & 2222.00 \\
Happiness $^{4}$ & $4.69(1.64)$ & $2.09(1.11)$ & $559.00^{\star * *}$ \\
Anger $^{4}$ & $0.31(0.59)$ & $1.31(1.03)$ & $959.00^{\star * *}$ \\
Sadness $^{4}$ & $0.36(0.52)$ & $1.33(1.29)$ & $1491.50^{\star * *}$ \\
Anxiety $^{4}$ & $0.11(0.22)$ & $0.94(1.15)$ & $1376.50^{\star * \star}$ \\
Disgust $^{4}$ & $0.08(0.26)$ & $0.77(1.04)$ & $1177.00^{\star * *}$ \\
Surprise $^{4}$ & $1.77(1.64)$ & $1.51(1.11)$ & 2843.50 \\
\hline
\end{tabular}

Note: The final column shows Mann-Whitney $U$ values to test group differences in emotion measures ( $n=81$ for the experimental group; $n=73$ for the control group). Significant $p$ values are indicated by asterisks $\left({ }^{* * *} p<.0001,{ }^{* *} p<.001\right)$. ${ }^{1}$ Scale range: -4 to 4 ; ${ }^{2}$ Significantly different from $0, p<.001 ;{ }^{3}$ Not significantly different from $0, p=.64 ;{ }^{4}$ Scale range: 0 to 8 . 
group vs. $M=2.09$ in the control group), followed by anger, sadness, and anxiety (Table 2). Importantly, participants of the control group felt neither pleasant nor unpleasant during the experiment, as indicated by their valence ratings, which did not differ from zero on a scale ranging from -4 to $4, p=.64$ ). Notably, neither valence nor arousal ratings increased or decreased during the course of the experiment, in either of the groups (the $\beta_{1}$ coefficients of linear regressions for mean valence and arousal ratings of each piece-calculated separately for each group across the course of the experiment-were less than .03). This shows that the mean valence and arousal values shown in Table 2 are representative across the entire experimental session.

\section{Discussion}

Music making positively changed mood, as indicated by a decrease of depression/anxiety, a decrease in fatigue, and an increase in vigor. The emotion ratings obtained during the sessions suggest that this positive change in mood was accompanied by an increase in positive emotion (as indicated by the happiness ratings), as well as by a decrease of negative emotion (as indicated by the anger, sadness, and anxiety ratings). It is unlikely that these effects were simply due to being in an experimental setting and performing an experiment with others, because in the control group the level of depression/anxiety (as measured by the first POMS scale) was not influenced by the experimental procedure.

It is also important to note that in the control group, the valence ratings of their own emotional state were neutral throughout the session (the valence ratings of the control group, i.e., the pleasantness ratings following the question "how did you feel during the last piece" were 0.1 on a scale from -4 to 4 ). Hence, the differences in mood measures between groups after the session (as compared to before the session) were not simply due to an aversive stimulus or procedure in the control group. In other words, the observation that participants in the control group judged their own emotional state as neither pleasant nor unpleasant throughout the session renders it unlikely that the positive mood effects in the music group merely emerged as a result of a potentially negative emotional impact of the experimental procedure in the control group. In this regard, it is also important to note that the pleasantness ratings were stable across the experiment in both groups. That is, individuals of the music group felt pleasant, and those of the control group neither pleasant nor unpleasant throughout the experiment.

Thus, the POMS data of the control group presumably reflect the normal change in mood after an hour of experimental testing during which the emotional valence of stimulus and experimental procedure is experienced as neutral by the participants (rendering it unlikely that the effects observed in the music group were simply due to a Hawthorne effect). However, the fact that fatigue and irritability increased, and vigor decreased in the control group makes the present control condition suboptimal, although the stimuli were matched on many parameters. Two methodological comments follow: First, a control condition selected for the likelihood that mood will not change will predictably differ from the music condition but might well fail to provide the same degree of experimental control. Second, using control conditions such as theatre playing, sports, playing games, painting, and the like, implies the problem that they are hardly well comparable to music making in terms of auditory perception, synchronization to an external beat, energy expenditure, and the like. Therefore, the question of whether it is the music, or a single aspect of the music making procedure that is not music specific, cannot satisfactorily be answered using such control conditions. In our study, we used a control procedure that was closely matched with the experimental procedure and experienced as neutral (i.e., neither as pleasant nor as unpleasant), the main difference between procedures being that in one group individuals made music and in the other group they did not.

Importantly, with regard to prospective use of the current method in music therapy, the depression/anxiety values (as measured with the POMS) did not change in the control group (but significantly decreased in the music group). That is, even though participants in the control condition were annoyed and fatigued by performing the experimental procedure, this irritation had no influence on the depression/anxiety scores.

The groups tended to differ at baseline with regard to the fatigue (which tended to be higher in the control group), but there were no baseline differences between groups on the depression/anxiety scale, and there was an interaction between groups and pre/post measures on all four scales. That is, even if groups were not identical at baseline, the increase in mood in the music group (as compared to the control group, and as compared with the pretest measures) was significant on all four scales. The differences in emotion ratings were present throughout the experiment (that is, also after the first piece); whether the length of the session has an influence on the change in mood measured directly after the session, and on longterm changes in mood (measured, for example, a day after the session) remains an open question.

We assume that the mood-increasing effects of the music making observed in the present study are at least 
partly due to the engagement in the social functions mentioned above ("The Seven Cs"), because only the music making (but not the control condition) involved social cognition, communication, and presumably also cooperation and social cohesion. However, we did not obtain data that could substantiate this assumption. Future studies could test this issue by comparing mood changes between individuals who make music in a group and individuals who make music alone. Likewise, it remains to be specified whether other social activities (such as playing games, theatre, sports, etc.) lead to similar changes in depression/anxiety (as observed in the present study), or other mood measures. As reviewed above, functional neuroimaging studies showed that music can modulate activity of all major limbic and paralimbic brain structures; that is, of structures crucially involved in the initiation, detection, generation, maintenance, termination, and modulation of emotions. Future studies could thus also investigate whether the method presented here results over the course of several weeks in functional and plastic changes (for example, changes in hippocampal volume) during the treatment of affective disorders such as depression, pathologic anxiety, and post-traumatic stress disorder (which are partly related to dysfunction of limbic structures such as the amygdala, the NAc, and the hippocampus, as well as of para-limbic structures such as the orbitofrontal cortex).

Notably, emotions also are closely linked to peripheralphysiological effects, i.e., emotions always have effects on the vegetative (or autonomic) nervous system, the hormonal (endocrine) system, and the immune system. Future studies could therefore also investigate effects of music making on these systems, perhaps even in patients with disorders related to dysfunctions and dysbalances within these systems.

\section{Author Note}

This work was supported by the German Research Foundation (Deutsche Forschungsgemeinschaft) through grant KO 2266/2-1. The research method introduced here was inspired by music workshops performed by Walter A. Siebel. Ronny Enk helped to make the auditory stimuli used for the neutral condition. Thomas Stegemann and Gunter Kreutz helped to improve this article considerably by providing valuable comments on an earlier version of this article.

Correspondence concerning this article should be addressed to Stefan Koelsch, Ph.D., Freie Universität Berlin, Habelschwerdter Allee 45, 14195 Berlin. E-MAIL: koelsch@cbs.mpg.de

\section{References}

Ball, T., Rahm, B., Eickhoff, S. B., Schulze-Bonhage, A., Speck, O., \& Mutschler, I. (2007). Response properties of human amygdala subregions: Evidence based on functional MRI combined with probabilistic anatomical maps. PLoS ONE, 2, e307.

Baumeister, R., \& Leary, M. R. (1995). The need to belong: Desire for interpersonal attachments as a fundamental human motivation. Psychological Bulletin, 117, 497-529.

Baumgartner, T., Lutz, K., Schmidt, C. F., \& JÄncke, L. (2006). The emotional power of music: How music enhances the feeling of affective pictures. Brain Research, 1075, 151-164.

Biehl, B., Dangel, S., \& Reiser, A. (1986). Profile of Mood States. Collegium Internationale Psychiatriae Scalarum. Internationale Skalen für Psychiatrie [International scales for psychiatry]. Weinheim, Germany: Beltz Test.

Bittman, B., Berk, L., Shannon, M., Sharaf, M., Westengard, J., Guegler, K. J., \& Ruff, D. W. (2005). Recreational music making modulates the human stress response: A preliminary individualized gene expression strategy. Medical Science Monitor, 11, 31-40.

Bittman, B., Bruhn, K. T., Stevens, C., Westengard, J., \& UMBACH, P. O. (2003). Recreational music-making: A cost-effective group interdisciplinary strategy for reducing burnout and improving mood states in long-term care workers. Advances in Mind Body Medicine, 19, 4-15.

Bittman, B. B., Snyder, C., Bruhn, K. T., Liebfreid, F., Stevens, C. K., Westengard, J., \& Umbach, P. O. (2004). Recreational music-making: An integrative group intervention for reducing burnout and improving mood states in first year associate degree nursing students: Insights and economic impact. International Journal of Nursing Education Scholarship, 1, 1044-1070.

Blood, A., \& Zatorre, R. J. (2001). Intensely pleasurable responses to music correlate with activity in brain regions implicated in reward and emotion. Proceedings of the National Academy of Sciences of the United States of America, 98, 11818-11823.

Bradley, M. M., \& Lang, P. J. (1994). Measuring emotion: The self-assessment manikin and the semantic differential. Journal of Behavior Therapy and Experimental Psychiatry, 25, 49-59.

Brown, S., \& Volgsten, U. (Eds.). (2006). Music and manipulation. On the social uses and social control of music. Oxford, UK: Berghahn.

Cacioppo, J. T., \& Hawkley, L. C. (2003). Social isolation and health, with an emphasis on underlying mechanisms. Perspectives in Biology and Medicine, 46, S39-S52. 
Callan, D. E., Tsytsarev, V., Hanakawa, T., Callan, A. M., Katsuhara, M., Fukuyama, H., \& Turner, B. (2006). Song and speech: Brain regions involved with perception and covert production. NeuroImage, 31, 1327-1342.

Cross, I., \& Morley, I. (2008). The evolution of music: Theories, definitions and the nature of the evidence. In S. Malloch \& C. Trevarthen (Eds.), Communicative musicality (pp. 61-82). Oxford, UK: Oxford University Press.

Dantzer, R., O’Connor, J. C., Freund, G. G., Johnson, R. W., \& Kelley, K. W. (2008). From inflammation to sickness and depression: When the immune system subjugates the brain. Nature Reviews Neuroscience, 9, 46-56.

Drevets, W. C., Price, J. L., Bardgett, M. E., Reich, T., Todd, R. D., \& Raichle, M. E. (2002). Glucose metabolism in the amygdala in depression: Relationship to diagnostic subtype and plasma cortisol levels. Pharmacology Biochemistry and Behavior, 71, 431-447.

Eldar, E., Ganor, O., Admon, R., Bleich, A., \& Hendler, T. (2007). Feeling the real world: Limbic response to music depends on related content. Cerebral Cortex, 17, 2828-2840.

Fitch, W. T. (2005). The evolution of music in comparative perspective. Annals of the New York Academy of Sciences, 1060, 29-49.

FITCH, W. T. (2006). The biology and evolution of music: A comparative perspective. Cognition, 100, 173-215.

Gosselin, N., Peretz, I., Johnsen, E., \& Adolphs, R. (2007). Amygdala damage impairs emotion recognition from music. Neuropsychologia, 45, 236-244.

Grape, C., Sandgren, M., Hansson, L. O., Ericson, M., \& Theorell, T. (2003). Does singing promote well-being? An empirical study of professional and amateur singers during a singing lesson. Integrative Psychological and Behavioral Science, 38, 65-74.

Harlow, H. F. (1958). The nature of love. American Psychologist, 13, 673-685.

Hillecke, T., Nickel, A., \& Bolay, H.V. (2005). Scientific perspectives on music therapy. Annals of the New York Academy of Sciences, 1060, 271-282.

House, J. S., Landis, K. R., \& Umberson, D. (1988). Social relationships and health. Science, 214, 540-545.

Huron, D. (2001). Is music an evolutionary adaptation? Annals of the New York Academy of Sciences, 930, 43-61.

JusLin, P. N., \& VÄSTFJÄLl, D. (2008). Emotional responses to music: The need to consider underlying mechanisms. Behavioral and Brain Science, 31, 559-575.

Kirschner, S., \& Tomasello, M. (2009). Joint drumming: Social context facilitates synchronization in preschool children. Journal of Experimental Child Psychology, 102, 299-314.

KoelsCH, S. (2005). Neural substrates of processing syntax and semantics in music. Current Opinion in Neurobiology, 15, 1-6.

Koelsch, S. (2010). Towards a neural basis of music-evoked emotions. Trends in Cognitive Sciences, 14, 131-137.
Koelsch, S., Fritz, T., von Cramon, D. Y., Müller, K., \& Friederici, A. D. (2006). Investigating emotion with music: An fMRI study. Human Brain Mapping, 27, 239-250.

Koelsch, S., Kasper, E., Sammler, D., Schulze, K., Gunter, T. C., \& Friederici, A. D. (2004). Music, language, and meaning: Brain signatures of semantic processing. Nature Neuroscience, 7, 511-514.

Koelsch, S., Remppis, A., Sammler, D., Jentschke, S., Mietchen, D., Fritz, T., Et Al. (2007). A cardiac signature of emotionality. European Journal of Neuroscience, 26, 33283338.

Koelsch S., Sammler, D., Jentschke, S., \& Siebel, W. A. (2008). EEG correlates of moderate intermittent explosive disorder. Clinical Neurophysiology, 119, 151-162.

Koelsch, S., \& Siebel, W. A. (2005). Towards a neural basis of music perception. Trends in Cognitive Sciences, 9, 578-584.

Koelsch, S., Siebel, W. A., \& Fritz, T. (2010). Functional neuroimaging. In P. Juslin \& J. A. Sloboda (Eds.), Music and emotion (pp. 313-346). Oxford: Oxford University Press.

Kreutz, G., Bongard, S., Rohrmann, S., Hodapp, V., \& Grebe, D. (2004). Effects of choir singing or listening on secretory immunoglobulin A, cortisol, and emotional state. Journal of Behavioral Medicine, 27, 623-635.

Kupfer, J., Brosig, B., \& Brähler, E. (2001). TorontoAlexithymie-Skala-26: Manual [Toronto alexithymia scale26: Manual]. Göttingen, Germany: Hogrefe Verlag.

Lerner, Y., Papo, D., Zhdanov, A., Belozersky, L., \& Hendler, T. (2009). Eyes wide shut: Amygdala mediates eyesclosed effect on emotional experience with music. PLoS One, 4, e6230.

Maratos, A. S., Gold, C., Wang, X., \& Crawford, M. J. (2008). Music therapy for depression. Cochrane database of systematic reviews, 1, CD004517. DOI: 10.1002/14651858. CD004517.pub2.

Mitterschiffthaler, M. T., Fu, C. H., Dalton, J. A., Andrew, C. M., \& Williams, S. C. (2007). A functional MRI study of happy and sad affective states evoked by classical music. Human Brain Mapping, 28, 1150-1162.

Overy, K., \& Molnar-SzaKacs, I. (2009). Being together in time: Musical experience and the mirror neuron system. Music Perception, 26, 489-504.

Patel, A. D. (2007). Music, language, and the brain. New York: Oxford University Press.

Patel, A. D., Iversen, J. R., Bregman, M. R., \& Schulz, I. (2009). Experimental evidence for synchronization to a musical beat in a nonhuman animal. Current Biology, 19, 827-830.

Quiroga Murcia, C., Bongard, S., \& Kreutz, G. (2009). Emotional and neurohumoral responses to dancing Tango Argentino: The effects of music and partner. Music and Medicine, 1, 14-21.

Quiroga Murcia, C., Kreutz, G., \& Bongard, S. (in press). Endokrine und immunologische Wirkungen von Musik 
[Endocrine and immunological effects of music]. In C. Schubert (Ed.), Psychoneuroimmunologie und Psychotherapie [Psychoneuroimmunology and psychotherapy]. Stuttgart, Germany: Schattauer.

Rilling, J., Gutman, D., Zeh, T., Pagnoni, G., Berns, G., \& Kilts, C. (2002). A neural basis for social cooperation. Neuron, 35, 395-405.

Singer, T., \& LAMm, C. (2009). The social neuroscience of empathy. Annals of the New York Academy of Sciences, 1156, 81-96.

Stein, M. B., Simmons, A. N., Feinstein, J. S., \& Paulus, M. P. (2007) Increased amygdala and insula activation during emotion processing in anxiety-prone subjects. American Journal of Psychiatry, 164, 318-27.

Steinbeis, N., \& Koelsch, S. (2008). Shared neural resources between music and language indicate semantic processing of musical tension-resolution patterns. Cerebral Cortex, 18, 1169-1178.

Steinbeis, N., \& Koelsch, S. (2009). Understanding the intentions behind manmade products elicits neural activity in areas dedicated to mental state attribution. Cerebral Cortex, 19, 619-623.
Tomasello, M., Carpenter, M., Call, J., Behne, T., \& Moll, H. (2005). Understanding and sharing intentions: The origins of cultural cognition. Behavioral and Brain Science, 28, 675-735.

Trenub, S. E. (2003). The developmental origins of musicality. Nature Neuroscience, 6, 669-673.

Valentine, E., \& Evans, C. (2001). The effects of solo singing, choral singing and swimming on mood and physiological indices. British Journal of Medical Psychology, 74, 115-120.

WALDON, E. G. (2001). The effects of group music therapy on mood states and cohesiveness in adult oncology patients. Journal of Music Therapy, 38, 212-238.

WARnER-SCHMidT, J. L., \& DUMAN, R. S. (2006). Hippocampal neurogenesis: Opposing effects of stress and antidepressant treatment. Hippocampus, 16, 239-249.

Wiltermuth, S. S., \& Heath, C. (2009). Synchrony and cooperation. Psychological Science, 20, 1-5.

Zatorre, R. J., Chen, J. L., \& Penhune, V. B. (2007). When the brain plays music: auditory-motor interactions in music perception and production. Nature Reviews Neuroscience, 8, 547-558.

\section{Appendix}

List of Stimuli

\begin{tabular}{lll}
\hline Composer & Title & ASIN Nr. \\
\hline Jonathan Richman & Egyptian Reggae & B00004U578 \\
Joel Perri & El Canto De Mi Antara & B0000284H1 \\
Georg Friedrich Händel & The Arrival of the Queen of Sheba & B0000057F6 \\
Jochen Schmidt-Hambrock & Roter Salon & B000025SS3 \\
Mike Post/ Pete Carpenter & Theme Song from Magnum, P.I. & B00030GMNQ \\
Rubén González & Enriqueta & B0010QCYGQ \\
Dordán & Annen Polka & B000002VXG \\
Joel Perri & Vuelta Vuelta & B00274OBVQ \\
Ron Goodwin & Theme from Miss Marple & B0029T2U6M \\
New Celtic Dimension & The Lucky Penny & B000003NHN \\
Johann Sebastian Bach & Badinerie (Ouvertüre No. 2, BWV 1067) & B001F67TQ6 \\
Joel Perri & Estudio Para Kena & B00274OBVQ \\
The Gas Band & An Angel Went Up in Flames & B000BEZQ18 \\
Rubén González & Fabiando & B0009GV2C0 \\
Georges Bizet & Prélude from Carmen & B000026A5L \\
Max Greger & Up to Date & B000077V11 \\
Louis Armstrong & St. Louis Blues & B000FBG0HG \\
Giacomo Rossini & Ouverture to Wilhelm Tell & B00000425N \\
\hline
\end{tabular}

